\title{
Saberes digitales en el profesorado universitario. Estudio en una escuela mexicana
}

\section{Digital knowledge in higher education teachers. Study in a Mexican school}

\author{
José Alejandro Lara-Rivera \\ Universidad Autónoma de Occidente. Sinaloa, México \\ alejandro.lara@uadeo.mx \\ Julio Cabero-Almenara \\ Universidad de Sevilla. Sevilla, España \\ cabero@us.es
}

\begin{abstract}
Resumen
Se presenta una investigación empírica que evalúa los saberes digitales en profesores de educación superior, los datos fueron recopilados en una institución mexicana ubicada en el noroeste del país. La muestra es probabilística aleatoria simple compuesta por 224 profesores clasificados desde las variables Edad, Grado académico y Género, ya que, se trabajó con el hipotético de que los conocimientos tecnológicos están relacionados con estos factores. Con la finalidad de buscar relación estadística en el agrupamiento de los datos, se realizó un factorial exploratorio por Kaiser-Meyer-Olkin (KMO) y prueba de esfericidad de Bartlett con el método de extracción de componentes principales y rotación varimax. Se aceptaron solo los factores con un autovalor superior a 1 y un peso factorial por ítem superior al 0.40 . Posteriormente se realizaron pruebas estadísticas no paramétricas para identificar relaciones desde las variables de cruce. Los hallazgos dan cuenta de que la Edad de los profesores es un factor diferenciador para la apropiación de saberes digitales.
\end{abstract}

Palabras clave: Saberes digitales, Educación superior, TIC, Alfabetización informacional, Formación de docentes.

\begin{abstract}
An empirical research is presented that assesses digital knowledge in higher education teachers, the data was collected in a Mexican institution located in the northwest of the country. The sample is simple random probabilistic composed of 224 teachers classified according to the variables Age, Academic Degree and Gender, since the hypothetical was worked on that technological knowledge is related to these factors. In order to find a statistical relationship in the grouping of data, an exploratory factorial was performed by Kaiser-Meyer-Olkin (KMO) and Bartlett's sphericity test with the principal component extraction method and varimax rotation. Only factors with an eigenvalue greater than 1 and a factorial weight per item greater than 0.40 were accepted. Subsequently, non-parametric statistical tests were performed to identify relationships from the crossover variables. The findings show that the age of the teachers is a differentiating factor for the appropriation of digital knowledge.
\end{abstract}

Keywords: Digital knowledge, higher education, ICT, Information literacy, teacher training.

RED. Revista de Educación a Distancia. Núm. 66, Vol. 21. Artíc. 4, 30-Abril-2021

DOI: http://dx.doi.org/10.6018/red.447911 


\section{Introducción}

Indicar desde el comienzo que la presente investigación forma parte de un estudio más amplio referente a Evaluación de Saberes Digitales en Instituciones de Educación Superior (Lara \& Grijalva, 2018), que tuvo como objetivo en un primer momento, conocer los niveles de competencia mediática y digital de los estudiantes universitarios. Los resultados dieron cuenta de que el avance en la carrera incidía de manera parcial para la adquisición de dichos saberes, por lo que el curriculum escolar no era un factor diferenciador. Estudios similares, como el de Sansot (2020), concluyen que los escenarios actuales muestran estudiantes con saberes tecnológicos adquiridos en ambientes ajenos al contexto escolar. Esto debido, por un lado, a la fallida inserción de las TIC para la creación de contenido educativo, y, por otro lado, persiste una férrea resistencia al cambio por parte de los docentes (Del Valle et. al. 2016), quienes prefieren los métodos de enseñanza tradicionales, que les otorgan control y autoridad ante el grupo, autoridad que se ve amenazada ya que el alumno progresivamente va adquiriendo mayor importancia y protagonismo en la aportación de información y datos, y en la construcción del conocimiento en la red (Cabero, 2015).

Dominio en TIC que se hace más necesario si tomamos en cuenta que las instituciones educativas actuales requieren ir incorporándolas progresivamente, pues son una variable crítica en la sociedad del conocimiento. En consecuencia, se requiere que los docentes tengan un adecuado dominio de estas para su integración en los procesos de enseñanza-aprendizaje (Hatlevik, Throndsen, Loi \& Gudmundsdottir, 2018; Roig-Vila, Mengual-Andrés \& QuintoMedrano, 2015), así como no exclusivamente para apoyar las prácticas existentes sino más bien para transformarlas (Uerz, Volman \& Kral, 2018).

La intención de este trabajo es analizar la otra cara de la moneda, es decir, evaluar el nivel de competencia digital de los profesores de educación superior, ya que, el reto al que nos enfrentamos se centra precisamente en adaptar los procesos tradicionales de enseñanza aprendizaje a nuevas propuestas metodológicas, ya que, los cambios generados tras la irrupción de las TIC en todos los ámbitos de nuestra sociedad incluido, el educativo, conllevan nuevas necesidades formativas por parte del profesorado para dar respuesta a la actual sociedad del conocimiento (Ruiz-Cabezas, Medina, Pérez \& Media, 2020). Inmersas en una sociedad donde el conocimiento se convierte en un elemento central del desarrollo, las Universidades se enfrentan al desafío de realizar modificaciones en sus estructuras pedagógicas. Una de estas líneas de cambio, es la innovación de los procesos educativos mediados por tecnologías digitales (Del Valle et. al. 2016).

De igual manera, Hernández, Ramírez-Martinell y Cassany (2014), enfatizan sobre la importancia de reconocer el surgimiento de una nueva cultura digital, que obliga el replanteo del sector educativo en todos sus niveles. Si bien es cierto, los modelos educativos mediados por las TIC favorecen el trabajo colaborativo, pensamiento crítico, autonomía y adquisición de competencias digitales, su éxito va en dos vías, ya que depende tanto del estudiante como del profesor (Gonzáles \& Abad, 2020). La educación, vista como pilar para el desarrollo de cualquier sociedad, debe adaptarse continuamente a las particularidades de los individuos que la conforman. En tal, Grijalva y Lara (2019) comentan que las universidades juegan un 
papel preponderante para formar sujetos críticos y alfabetizados en entornos altamente digitales, pues son las IES espacios históricamente encargados de generar, transferir y aplicar nuevos conocimientos.

\section{El docente universitario y su actitud ante las TIC.}

La actitud de los docentes es un factor esencial para la inclusión de las TIC en los contextos educativos, pues a partir de una concepción positiva de los métodos, las ventajas del uso de herramientas versátiles y los beneficios pedagógicos, se dedicará más tiempo al diseño de actividades orientadas en este sentido. Para Grijalva y Moreno (2017), educar en medios implica un cambio de actitud docente para la incorporación de la tecnología a las estrategias pedagógicas y a la evaluación de los saberes digitales. De igual manera, Cuban (2001) asegura que las creencias y actitudes de los profesores acerca de cómo aprenden los alumnos, condicionan en gran parte la inclusión de las TIC en su práctica docente. Cabero (2020), comenta que en algunos sectores educativos se ha creado una imagen sobre la formación virtual, presentándola como una formación de segunda categoría respecto a la presencial. Solo cuando el docente haya reflexionado sobre el uso particular que les da a las TIC en sus prácticas educativas, podrá iniciar un proceso de formación eficiente a través de tutoriales, videos, MOOC, webinars, talleres, etc. (UNESCO, 2016).

Esta actitud y creencia de los docentes viene determinada por una serie de variables, siendo la edad, una de las que ha demostrado incidir fuertemente, encontrándonos que por lo general los docentes de mayor edad y con mayor experiencia docente, poseen un perfil de formación en TIC mucho más bajo que aquellos que son más jóvenes, quienes suelen tener más competencias instrumentales y didácticas para la incorporación de las TIC (Fernández-Cruz \& Fernández-Díaz, 2016; Gallardo, Poma \& Esteve, 2018; Garzón, Sola, Ortega, Marín \& Gómez, 2020; Lázaro, Usart \& Gisbert, 2019; Solís \& Jara, 2019; López, Pozo, Fuentes \& Romero, 2019; Infante-Moro, Infante-Moro \& Gallardo, 2021).

Por lo que se refiere a otra de las variables que se considerarán en el presente trabajo: el género. Los estudios no aportan una visión definitiva, así un grupo de investigadores encontraron un efecto positivo en el género masculino (Cai et al., 2017), mientras que, Siddiq y Scherer (2019) en un meta análisis con un recuento de 46 tamaños de efectos de 23 estudios empíricos, encontraron que las diferencias de género eran más positivas y significativas en el género femenino.

Esto último está muy alineado a lo que abordamos en esta investigación, ya que hemos partido del hipotético de que los saberes digitales de los docentes están relacionados a la Edad, Grado académico y Género.

\section{Estándares TIC para profesores de educación superior.}

Diversos han sido los estándares propuestos para analizar las competencias específicas que conllevan la competencia digital del docente (Durán, Gutiérrez \& Prendes, 2016; Cabero \& Martínez, 2019; Lázaro, Usart \& Gisbert, 2019; Rodríguez-García, Raso Sánchez \& Ruiz- 
Palmero, 2019; Silva, Morales, Lázaro \& Gisbert, 2019; Cabero \& Palacios, 2020), destacan de todos ellos los siguientes:

- Ministerio de Educación de Chile (2006); área pedagógica, aspectos sociales, éticos y legales, aspectos técnicos, gestión escolar y desarrollo profesional.

- The Educational Testing Service (ETS, 2007); acceder, administrar, integrar, evaluar y crear.

- The International Society for Technology in Education (ISTE, 2008); facilitar e inspirar el aprendizaje y la creatividad, diseñar y desarrollar experiencias y evaluaciones del aprendizaje, modelo de trabajo y aprendizaje en la era digital, ciudadanía digital, participar en el desarrollo profesional y el liderazgo.

- Organización de las Naciones Unidas para la Educación, la Ciencia y la Cultura (UNESCO, 2011); alfabetización tecnológica, profundización del conocimiento y creación del conocimiento.

- Instituto Nacional de Tecnologías Educativas y de Formación del Profesorado (INTEF, 2017); información y alfabetización informacional, comunicación y colaboración, creación de contenidos digitales, seguridad y resolución de problemas.

- The digital competence framework for citizens, DigComEdu (Comisión Europea, 2017); compromiso profesional, recursos digitales, enseñanza y aprendizaje, evaluación, empoderar a los estudiantes y facilitar la competencia digital de los estudiantes.

Indicar que de dichas propuestas sirvieron para la elaboración de los ítems del instrumento utilizado en la investigación, así como para la elaboración de una taxonomía conceptual que expresa de manera cercana el contexto mexicano y en este caso se ha adaptado de manera particular a la Universidad Autónoma de Occidente (UAdeO), con las siguientes dimensiones: 1) Literacidad digital; 2) Creación y socialización de contenido digital; 3) Seguridad digital; 4) Ciudadanía digital; y 5) Desarrollo profesional.

Literacidad digital: Esta dimensión integra conocimientos, habilidades y actitudes dirigidas a la búsqueda efectiva de contenido digital y a su manejo, mediante la consideración de palabras clave y metadatos; adopción de una postura crítica (consulta en bases de datos especializadas, realización de búsquedas avanzadas); aplicación de estrategias determinadas (uso de operadores booleanos, definición de filtros); y consideraciones para un manejo adecuado de la información (referencias, difusión y comunicación).

Creación y Socialización de contenido digital: Se refiere a la capacidad para trabajar con archivos de texto, bases de datos y archivos multimedia, incluyendo fotografía, audio y video. De acuerdo a la literatura de educación digital, el manejo de contenidos digitales representa uno de los nuevos pilares en la formación universitaria, en este sentido, el profesor universitario busca desarrollar competencias para la producción, reproducción y edición de contendidos de distintas plataformas digitales. El principio de la socialización y comunicación en entornos digitales se basa las premisas del pensamiento pedagógico relacional donde se parte de la idea de que los sujetos construyen conocimiento a partir de las interacciones que establezcan en sus entornos personales de aprendizaje.

Saberes digitales en el profesorado universitario. Estudio en una escuela mexicana. José Alejandro Lara-Rivera y Julio Cabero-Almenara.

Página 4 de 19 
Seguridad digital: Esta dimensión tiene que ver con la protección de información y datos personales, protección de la identidad digital, y uso responsable y seguro de entornos digitales.

Ciudadanía digital: Aquí se analiza lo concerniente a conocimientos, valores, actitudes y habilidades sobre los usos sociales, comportamientos éticos, respeto a la propiedad intelectual, integridad de datos, difusión de información sensible y a las normas relativas a los derechos y deberes de los usuarios de sistemas digitales en el espacio público, específicamente en el contexto escolar. Asimismo, la ciudadanía digital se relaciona con algunas reglas sobre el comportamiento y el buen uso de las tecnologías. En lo anterior, la discusión epistemológica sobre ciudadanía en educación representa un campo amplio sobre el papel de la Universidad en la formación de ciudadanos, en ello la ciudadanía digital es una mirada integradora de tales perspectivas que incorpora la categoría de lo digital como elemento para constituir al individuo, es decir, los ciudadanos ya no son únicamente sujetos de un espacio físico, sino que interactúan en entornos globales e interconectados.

Desarrollo profesional: Por último, la dimensión de Desarrollo Profesional se refiere al uso que los docentes hacen de las TIC como medio de especialización y desarrollo profesional, informándose y accediendo a diversas fuentes para mejorar sus prácticas y facilitando el intercambio de experiencias que contribuyan mediante un proceso de reflexión con diversos actores educativos, a conseguir mejores procesos de enseñanza y aprendizaje.

\section{Método}

Para realizar el análisis, se trabajó con el programa estadístico SPSS versión 21 y se construyeron las variables EDAD, dividida en cinco grupos: 24-29, 30-39, 40-49, 50-59, 60-70; GRADO, dividida en tres grupos: licenciatura, maestría, doctorado; y GÉNERO, dividida en dos grupos: hombre; mujer. Lo anterior con la intención de encontrar los factores que inciden en la apropiación de los saberes digitales de los profesores de la Universidad Autónoma de Occidente.

El cuestionario está estructurado tipo escala Likert a 5 puntos, donde 1 representa al lado negativo de la escala y el 5 al lado positivo. El instrumento contempla 59 ítems que evalúan las dimensiones descritas anteriormente: Literacidad digital (14 ítems); Creación y socialización de contenido digital (12 ítems); Seguridad digital (12 ítems); Ciudadanía digital (12 ítems) y Desarrollo profesional (9 ítems). Se aplicó en formato digital autocumplimentado mediante computadoras, teléfonos celulares y tabletas electrónicas. El levantamiento de la información duró tres semanas. La muestra es probabilística aleatoria simple compuesta por 224 profesores de la UAdeO, Unidad Regional Culiacán, vigentes al 2020. La fiabilidad y consistencia interna del instrumento (59 ítems) arroja un Alfa de Cronbach favorable con valor de 0.942. El alcance de la investigación es descriptivo, exploratorio y relacional, al mostrar un panorama sobre los saberes digitales y vincularse desde la Edad, Grado académico y Género de los docentes. Se trabajó con la hipótesis de que

Saberes digitales en el profesorado universitario. Estudio en una escuela mexicana. José Alejandro Lara-Rivera y Julio Cabero-Almenara.

Página 5 de 19 
dichas variables inciden en los saberes digitales de los profesores, por lo que puede ser expresada de la siguiente manera:

Hipótesis nula (H0): No hay diferencias significativas entre los niveles de saberes digitales y las variables edad, grado y género del profesorado, con un riesgo alfa de equivocarnos del .05.

Hipótesis alternativa (H1): Si hay diferencias significativas entre los niveles de saberes digitales y las variables edad, grado y género del profesorado, con un riesgo alfa de equivocarnos del .05.

Con la finalidad de buscar relación estadística en el agrupamiento de los datos, se realizó un análisis factorial exploratorio por Kaiser-Meyer-Olkin (KMO) y prueba de esfericidad de Bartlett con el método de extracción de componentes principales y rotación varimax. Se aceptaron solo los factores con un autovalor superior a 1 y un peso factorial por ítem superior al 0.40. Posteriormente se realizaron pruebas estadísticas no paramétricas para identificar relaciones desde las variables de cruce.

\section{Resultados}

Los hallazgos dan cuenta de que los profesores en general tienen saberes digitales aceptables, aunque con tendencia a regular, ya que, el 12,1\% se encuentra en el nivel competencia baja, el $31.7 \%$ en nivel regular, $41.1 \%$ en nivel aceptable y $15.2 \%$ en nivel avanzado.

Cuadro 1. Evaluación general sobre saberes digitales (Fuente de elaboración propia).

\begin{tabular}{|ll|r|r|r|r|}
\hline & Frecuencia & Porcentaje & $\begin{array}{c}\text { Porcentaje } \\
\text { válido }\end{array}$ & $\begin{array}{c}\text { Porcentaje } \\
\text { acumulado }\end{array}$ \\
\hline Válidos & BAJO & 27 & 12.1 & 12.1 & 12.1 \\
& REGULAR & 71 & 31.7 & 31.7 & 43.8 \\
& ACEPTABLE & 92 & 41.1 & 41.1 & 84.8 \\
& AVANZADO & 34 & 15.2 & 15.2 & 100.0 \\
& Total & 224 & 100.0 & 100.0 & \\
\hline
\end{tabular}

Elaboración propia con SPSS V21

Al mismo tiempo los profesores señalan tener una baja capacitación respecto a las TIC. La siguiente gráfica muestra el resultado obtenido al preguntar a los profesores respecto a si han participado en los últimos 5 años en algún congreso, seminario o taller referente a innovación educativa y TIC. De los 228 sujetos, 122 (53.5\%) respondieron no haber tenido participación en este tipo de actividades. 
Gráfica 1. Ha participado en los últimos 5 años en algún congreso, seminario o taller referente a innovación educativa y TIC? (Fuente: elaboración propia).

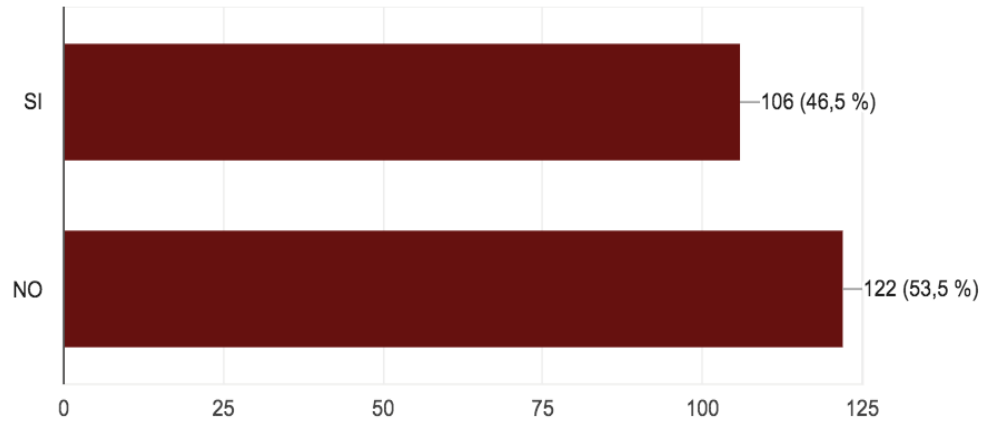

En primer lugar, analizaremos los índices de fiabilidad de cada una de los factores y la adecuación de los diferentes ítems a los factores indicados en el trabajo. Para ello se realizó un análisis factorial exploratorio y se encontró que los 59 ítems podían ser organizados de manera distinta en función de la correlación de las respuestas de los sujetos, quedando la asociación tal como se presenta en el cuadro $\mathrm{n}^{\mathbf{0}} 2$.

Cuadro 2. Factorización de dimensiones (Fuente: Elaboración propia).

\begin{tabular}{|c|c|c|c|c|c|}
\hline Dimensiones & Factores & Identificador & No. de ítems & KMO & Bartlet \\
\hline Literacidad digital & $\begin{array}{l}\text { * Pensamiento crítico } \\
\text { * Literacidad } \\
\text { * Búsqueda efectiva }\end{array}$ & $\begin{array}{l}\text { PensCrit } \\
\text { Literacidad } \\
\text { BusqEfect }\end{array}$ & $\begin{array}{l}5 \text { ítems } \\
4 \text { ítems } \\
5 \text { ítems }\end{array}$ & 0.854 & 0.000 \\
\hline $\begin{array}{c}\text { Creación y socialización de } \\
\text { contenido digital }\end{array}$ & $\begin{array}{l}\text { * Socialización } \\
\text { * Creación } \\
\end{array}$ & $\begin{array}{l}\text { Socializacion } \\
\text { Creacion }\end{array}$ & $\begin{array}{l}7 \text { ítems } \\
5 \text { ítems }\end{array}$ & 0.873 & 0.000 \\
\hline Seguridad digital & $\begin{array}{l}* \text { Seguridad } \\
* \text { Legalidad } \\
\end{array}$ & $\begin{array}{l}\text { Seguridad } \\
\text { Legalidad }\end{array}$ & $\begin{array}{l}7 \text { ítems } \\
5 \text { ítems }\end{array}$ & 0.802 & 0.000 \\
\hline Ciudadanía digital & $\begin{array}{l}\text { * Ética digital } \\
* \text { Identidad digital } \\
* \text { Compromiso social }\end{array}$ & $\begin{array}{l}\text { EticaDig } \\
\text { IdentDig } \\
\text { ComprSocial }\end{array}$ & $\begin{array}{l}5 \text { ítems } \\
5 \text { ítems } \\
2 \text { ítems }\end{array}$ & 0.676 & 0.000 \\
\hline Desarrollo profesional & $\begin{array}{l}\text { * Autonomía } \\
\text { * Desarrollo profesional }\end{array}$ & $\begin{array}{l}\text { Autonomia } \\
\text { DesarrProf }\end{array}$ & $\begin{array}{l}5 \text { ítems } \\
4 \text { ítems }\end{array}$ & 0.855 & 0.000 \\
\hline
\end{tabular}

A continuación, se detalla la factorización de cada una de las dimensiones, y los ítems que las conformarán.

Cuadro 3. Factorización de la dimensión Literacidad Digital (Fuente: Elaboración propia).

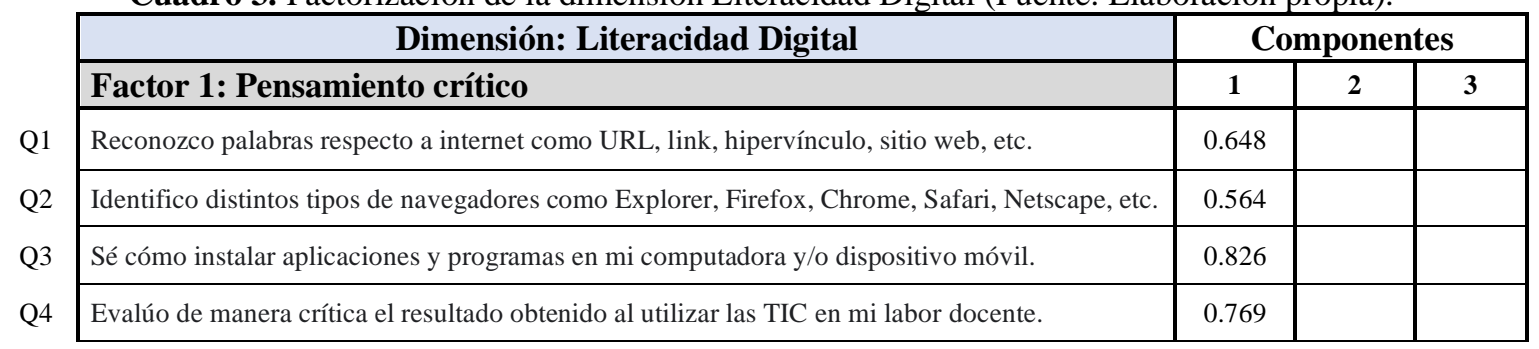

Saberes digitales en el profesorado universitario. Estudio en una escuela mexicana. José Alejandro Lara-Rivera y Julio Cabero-Almenara. 
RED. Revista de Educación a Distancia. Núm. 66, Vol. 21. Artíc. 4, 30-Abril -2021

DOI: http://dx.doi.org/10.6018/red.447911

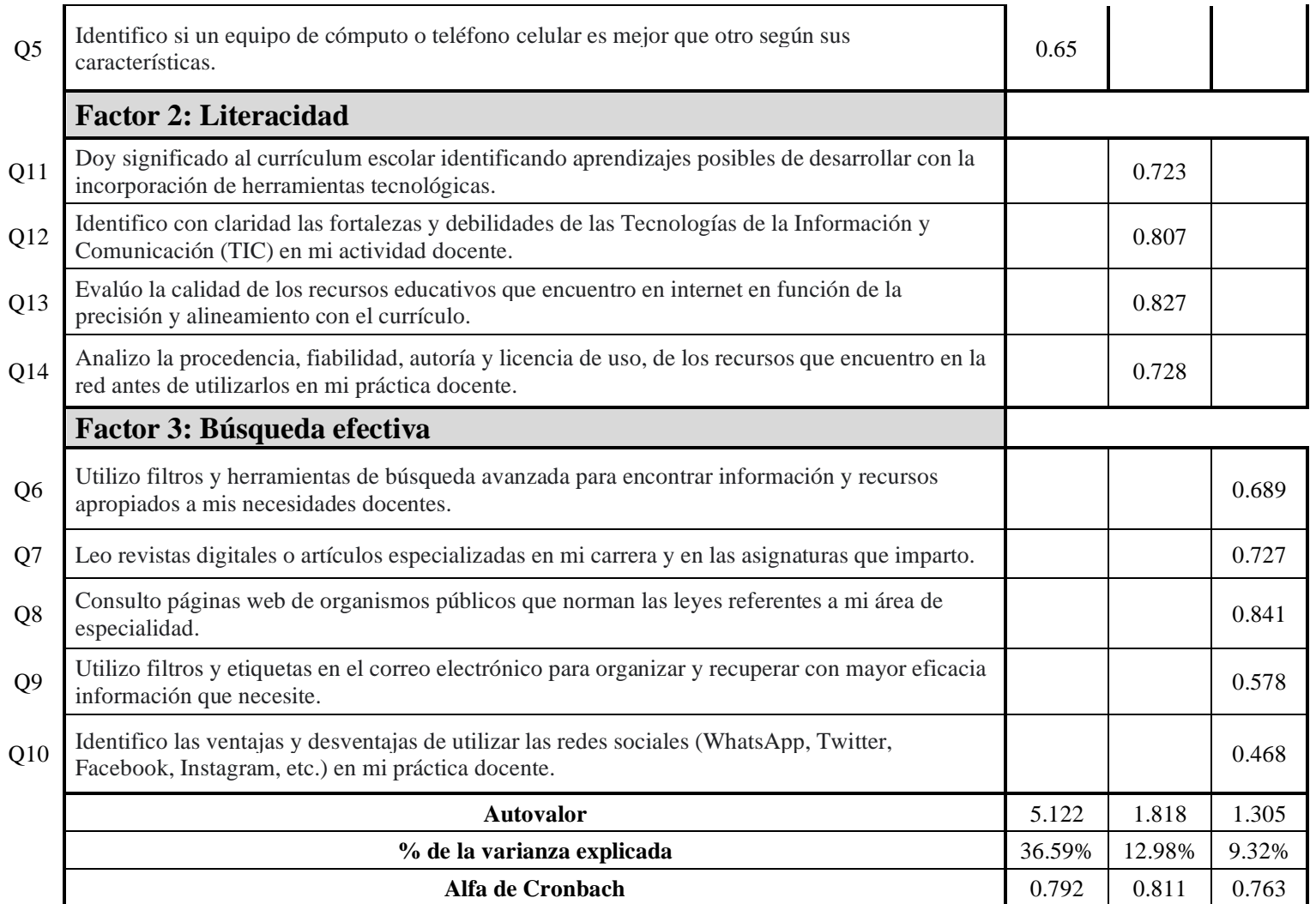

Cuadro 4. Factorización de la dimensión Creación y Socialización. (Fuente: Elaboración propia).

\begin{tabular}{|c|c|c|c|}
\hline & \multirow{2}{*}{$\begin{array}{l}\text { Dimensión: Creación y Socialización } \\
\text { Factor 1: Socialización }\end{array}$} & \multicolumn{2}{|c|}{ Componentes } \\
\hline & & 1 & 2 \\
\hline Q20 & Diseño actividades online que complementan el proceso de enseñanza aprendizaje presencial. & 0.732 & \\
\hline Q21 & Utilizo plataformas digitales que promuevan el trabajo colaborativo en red con alumnos y profesores. & 0.759 & \\
\hline Q22 & $\begin{array}{l}\text { Participo de manera colaborativa en la creación de recursos educativos digitales con otros compañeros } \\
\text { docentes. }\end{array}$ & 0.635 & \\
\hline Q23 & $\begin{array}{l}\text { Utilizo los servicios de internet para apoyar las tareas administrativas propias de mi actividad docente } \\
\text { como, tutorías, reportes, etc. }\end{array}$ & 0.737 & \\
\hline Q24 & $\begin{array}{l}\text { Utilizo con fines académicos el correo electrónico y redes sociales, como instrumento habitual de } \\
\text { comunicación con alumnos y profesores. }\end{array}$ & 0.476 & \\
\hline Q25 & Participo en temas académicos o de interés social en espacios abiertos como Twitter o Facebook. & 0.601 & \\
\hline \multirow[t]{2}{*}{ Q26 } & $\begin{array}{l}\text { Busco nuevas herramientas de comunicación en línea con el objetivo de mejorar los procesos de } \\
\text { enseñanza aprendizaje. }\end{array}$ & 0.731 & \\
\hline & Factor 2: Creación & & \\
\hline Q15 & Sé crear, abrir, guardar y editar archivos de office (Word, excel, PowerPoint...) & & 0.742 \\
\hline Q16 & Sé crear y subir un video a YouTube. & & 0.551 \\
\hline Q17 & Elaboro materiales y recursos educativos digitales incorporando imágenes, video, texto, etc. & & 0.648 \\
\hline Q18 & $\begin{array}{l}\text { Integro, combino y modifico contenido digital encontrado en la Red ajustándolo a mis necesidades y } \\
\text { respetando licencias de uso. }\end{array}$ & & 0.624 \\
\hline \multirow[t]{2}{*}{ Q19 } & Puedo elaborar mapas mentales con programas como CmapTools, Freemind, entre otros. & & 0.734 \\
\hline & Autovalor & 4.855 & 1.297 \\
\hline
\end{tabular}

Saberes digitales en el profesorado universitario. Estudio en una escuela mexicana. José Alejandro Lara-Rivera y Julio Cabero-Almenara. 
RED. Revista de Educación a Distancia. Núm. 66, Vol. 21. Artíc. 4, 30-Abril -2021

DOI: http://dx.doi.org/10.6018/red.447911

\begin{tabular}{|c|c|c|}
\hline \% de la varianza explicada & $40.46 \%$ & $10.81 \%$ \\
\hline Alfa de Cronbach & 0.769 & 0.730 \\
\hline
\end{tabular}

Cuadro 5. Factorización de la dimensión Seguridad digital. (Fuente: Elaboración propia).

\begin{tabular}{|c|c|c|}
\hline Dimensión: Seguridad Digital & \multicolumn{2}{|c|}{ Componentes } \\
\hline Factor 1: Seguridad & $\mathbf{1}$ & 2 \\
\hline Sé cómo eliminar un virus de mi equipo. & 0.807 & \\
\hline Sé cómo instalar en mis dispositivos software de protección o firewall. & 0.845 & \\
\hline Sé cómo realizar copias de seguridad de mis archivos y carpetas. & 0.752 & \\
\hline Utilizo la nube como herramienta de almacenamiento de información importante. & 0.694 & \\
\hline Genero claves y contraseñas seguras que combinen números, signos y letras. & 0.457 & \\
\hline Utilizo diferentes contraseñas para las aplicaciones digitales que manejo. & 0.455 & \\
\hline Utilizo candados para bloquear contenido ilegal, inapropiado o poco confiable de internet. & 0.465 & \\
\hline \multicolumn{3}{|l|}{ Factor 2: Legalidad } \\
\hline Identifico información relevante evaluando las distintas fuentes y su procedencia. & & 0.53 \\
\hline Soy consciente de los riesgos que tiene proporcionar información personal por internet. & & 0.554 \\
\hline $\begin{array}{l}\text { Utilizo sistemas de protección (antivirus, corta fuegos...) para garantizar y asegurar la protección } \\
\text { técnica del equipo. }\end{array}$ & & 0.522 \\
\hline Leo los términos y condiciones de las aplicaciones que descargo. & & 0.727 \\
\hline $\begin{array}{l}\text { Reconozco los aspectos éticos y legales asociados a la información digital tales como privacidad, } \\
\text { propiedad intelectual y seguridad de la información. }\end{array}$ & & 0.817 \\
\hline Autovalor & 4.346 & 1.628 \\
\hline$\%$ de la varianza explicada & $36.22 \%$ & $13.57 \%$ \\
\hline Alfa de Cronbach & 0.809 & 0.702 \\
\hline
\end{tabular}

Cuadro 6. Factorización de la dimensión Ciudadanía digital. (Fuente: Elaboración propia).

\begin{tabular}{|c|c|c|c|}
\hline Dimensión: Ciudadanía Digital & \multicolumn{3}{|c|}{ Componentes } \\
\hline Factor 1: Ética digital & 1 & 2 & 3 \\
\hline Cuido mi imagen y reputación digital. & 0.585 & & \\
\hline Soy selectivo con las personas que agrego a mis redes sociales. & 0.53 & & \\
\hline Valido que la información que comparto en redes sociales sea verídica. & 0.632 & & \\
\hline $\begin{array}{l}\text { Promuevo con los alumnos la utilización de contenido digital respetando en todo tiempo los } \\
\text { derechos de autor. }\end{array}$ & 0.818 & & \\
\hline $\begin{array}{l}\text { Reviso que los estudiantes no incurran en situaciones de plagio o fraude en sus trabajos } \\
\text { escolares. }\end{array}$ & 0.714 & & \\
\hline \multicolumn{4}{|l|}{ Factor 2: Identidad digital } \\
\hline Utilizo un mismo nombre de usuario que me identifique en distintas redes sociales. & & 0.453 & \\
\hline $\begin{array}{l}\text { Soy miembro de alguna asociación o red académica que aborde temas sobre ciudadanía } \\
\text { digital. }\end{array}$ & & 0.711 & \\
\hline $\begin{array}{l}\text { Selecciono y comparto con otros docentes documentación sobre los peligros y usos perversos } \\
\text { de internet. }\end{array}$ & & 0.788 & \\
\hline Utilizo las TIC para generar conciencia social sobre temas relevantes. & & 0.664 & \\
\hline Identifico con facilidad información tendenciosa que circula en redes sociales. & & 0.451 & \\
\hline
\end{tabular}

Saberes digitales en el profesorado universitario. Estudio en una escuela mexicana. José Alejandro Lara-Rivera y Julio Cabero-Almenara. 
RED. Revista de Educación a Distancia. Núm. 66, Vol. 21. Artíc. 4, 30-Abril -2021

DOI: http://dx.doi.org/10.6018/red.447911

\begin{tabular}{c|l|c|c|c|}
\multicolumn{2}{|l|}{ Factor 3: Compromiso social } & & & 0.973 \\
\cline { 2 - 5 } Q42 & $\begin{array}{l}\text { Evito compartir archivos que promuevan violencia, pornografía, bullying u otros factores que } \\
\text { afectan mi identidad digital. }\end{array}$ & & & 0.964 \\
\cline { 2 - 5 } & $\begin{array}{l}\text { Evito difundir texto, imágenes o videos sexistas, racistas o que promuevan algún tipo de } \\
\text { discriminación. }\end{array}$ & 3.175 & 1.969 & 1.388 \\
\hline \multicolumn{1}{|c|}{ Autovalor } & $26.45 \%$ & $16.41 \%$ & $11.56 \%$ \\
\hline \% de la varianza explicada & 0.708 & 0.634 & 0.979 \\
\hline
\end{tabular}

Cuadro 7. Factorización de la dimensión Desarrollo profesional. (Fuente: Elaboración propia).

\begin{tabular}{|c|c|c|c|}
\hline & \multirow{2}{*}{$\begin{array}{l}\text { Dimensión: Desarrollo Profesional } \\
\text { Factor 1: Autonomía }\end{array}$} & \multicolumn{2}{|c|}{ Componentes } \\
\hline \multirow[b]{2}{*}{ Q51 } & & 1 & 2 \\
\hline & Soy capaz de resolver problemas técnicos que se presentan en el aula. & 0.825 & \\
\hline Q52 & $\begin{array}{l}\text { Identifico las ventajas e inconvenientes del uso de los dispositivos, herramientas, entornos y } \\
\text { servicios digitales que utilizo de forma habitual en mi labor docente. }\end{array}$ & 0.774 & \\
\hline Q53 & Aprendo de forma autónoma el uso de herramientas y aplicaciones digitales. & 0.848 & \\
\hline Q54 & Utilizo fuentes diversas de información para mi actualización en TIC. & 0.701 & \\
\hline \multirow[t]{2}{*}{ Q59 } & $\begin{array}{l}\text { Como docente universitario, soy consciente de la necesidad de formación en herramientas digitales } \\
\text { aplicadas a la educación superior. }\end{array}$ & 0.410 & \\
\hline & \multicolumn{3}{|l|}{ Factor 2: Desarrollo profesional } \\
\hline Q55 & Participo en actividades formativas relacionadas con el uso de las TIC. & & 0.56 \\
\hline Q56 & $\begin{array}{l}\text { Recurro habitualmente a plataformas y repositorios de recursos digitales para mejorar mis } \\
\text { competencias en TIC. }\end{array}$ & & 0.744 \\
\hline Q57 & Mantengo un listado de sitios relevantes a mi quehacer docente y desarrollo profesional. & & 0.836 \\
\hline \multirow[t]{4}{*}{ Q58 } & $\begin{array}{l}\text { Evalúo y selecciono nuevas fuentes de información e innovaciones tecnológicas como fundamento } \\
\text { para la adecuación a mi práctica docente. }\end{array}$ & & 0.787 \\
\hline & Autovalor & 4.409 & 1.19 \\
\hline & $\%$ de la varianza explicada & $48.99 \%$ & $13.22 \%$ \\
\hline & Alfa de Cronbach & 0.828 & 0.801 \\
\hline
\end{tabular}

Los resultados señalan dos aspectos, en primer lugar, unos altos niveles de fiabilidad para cada uno de los factores de acuerdo con Mateo (2004) y en segundo término una adecuada incorporación de los ítems del instrumento en los diferentes factores identificados en nuestro estudio.

Realizado este contraste de fiabilidad del instrumento, se pasará al análisis de las hipótesis formuladas, referidas a la posible relación, o no, entre los niveles de saberes digitales y las variables edad, grado académico y género. Indicar en primer lugar que se aplicó la prueba de normalidad Kolmogorov-Smirnov (Siegel, 1976), para determinar la utilización de una prueba paramétrica o no paramétrica, y en función del resultado encontrado determinar el estadístico que se aplicaría.

Saberes digitales en el profesorado universitario. Estudio en una escuela mexicana. José Alejandro Lara-Rivera y Julio Cabero-Almenara. 
RED. Revista de Educación a Distancia. Núm. 66, Vol. 21. Artíc. 4, 30-Abril -2021

DOI: http://dx.doi.org/10.6018/red.447911

Por lo que se refiere a la edad, los valores de la prueba de Kolmogorov-Smirnov, se presentan en el cuadro $\mathrm{n}^{\circ} 8$.

Cuadro 8. Prueba de normalidad (Edad / 12 Factores) (Fuente: Elaboración propia).

\begin{tabular}{|c|c|c|c|c|c|c|c|c|}
\hline \multicolumn{9}{|c|}{ Pruebas de normalidad (Edad / Factores) } \\
\hline \multicolumn{9}{|c|}{ Kolmogorov-Smirnov } \\
\hline Factor & Edad & Sig. & Factor & Edad & Sig. & Factor & Edad & Sig. \\
\hline \multirow{5}{*}{ PensCrit } & $24-29$ & 0.040 & \multirow{5}{*}{ Creacion } & $24-29$ & 0.006 & \multirow{5}{*}{ IdenDig } & $24-29$ & 0.033 \\
\hline & $30-39$ & 0.012 & & $30-39$ & 0.001 & & $30-39$ & 0.049 \\
\hline & $40-49$ & 0.000 & & $40-49$ & 0.000 & & $40-49$ & 0.004 \\
\hline & $50-59$ & $.200^{\circ}$ & & $50-59$ & $.200^{*}$ & & $50-59$ & 0.077 \\
\hline & $60-70$ & $.200^{\circ}$ & & $60-70$ & $.200^{\circ}$ & & $60-70$ & $.200^{\circ}$ \\
\hline \multirow{5}{*}{ Literacidad } & $24-29$ & 0.001 & \multirow{5}{*}{ Seguridad } & $24-29$ & $.200^{\circ}$ & \multirow{5}{*}{ ComprSocial } & $24-29$ & 0.000 \\
\hline & $30-39$ & 0.000 & & $30-39$ & $.200^{\circ}$ & & $30-39$ & 0.000 \\
\hline & $40-49$ & 0.000 & & $40-49$ & 0.015 & & $40-49$ & 0.000 \\
\hline & $50-59$ & 0.048 & & $50-59$ & 0.067 & & $50-59$ & 0.000 \\
\hline & $60-70$ & 0.075 & & $60-70$ & 0.008 & & $60-70$ & 0.000 \\
\hline \multirow{5}{*}{ BusqEfect } & $24-29$ & 0.003 & \multirow{5}{*}{ Legalidad } & $24-29$ & 0.004 & \multirow{5}{*}{ Autonomia } & $24-29$ & 0.127 \\
\hline & $30-39$ & 0.002 & & $30-39$ & 0.003 & & $30-39$ & 0.000 \\
\hline & $40-49$ & 0.007 & & $40-49$ & 0.000 & & $40-49$ & 0.000 \\
\hline & $50-59$ & $.200^{\circ}$ & & $50-59$ & 0.006 & & $50-59$ & 0.076 \\
\hline & $60-70$ & $.200^{\circ}$ & & $60-70$ & 0.140 & & $60-70$ & 0.103 \\
\hline \multirow{5}{*}{ Socializacion } & $24-29$ & $.200^{\circ}$ & \multirow{5}{*}{ EticaDig } & $24-29$ & 0.065 & \multirow{5}{*}{ DesarrProf } & $24-29$ & $.200^{\circ}$ \\
\hline & $30-39$ & 0.000 & & $30-39$ & 0.000 & & $30-39$ & 0.072 \\
\hline & $40-49$ & 0.019 & & $40-49$ & 0.000 & & $40-49$ & 0.097 \\
\hline & $50-59$ & 0.157 & & $50-59$ & 0.000 & & $50-59$ & 0.012 \\
\hline & $60-70$ & 0.049 & & $60-70$ & 0.001 & & $60-70$ & 0.041 \\
\hline
\end{tabular}

Los valores alcanzados indican, que la distribución de los datos (Edad / Factores) es anormal, por lo que se utilizarán pruebas no paramétricas para el análisis, en concreto el estadístico de Kruskal - Wallis (Siegel, 1976), valores que se presentan en el cuadro ${ }^{\circ} 9$.

Cuadro 9. Prueba de Kruskal-Wallis (Edad) (Fuente: Elaboración propia).

\begin{tabular}{l|r|r|r|r|r|r|r|r|r|r|r|r|}
\hline & PensCrit & Literacidad & BusqEfect & Socializacion & Creacion & Seguridad & Legalidad & EticaDig & IdentDig & ComprSocial & Autonomia & DesarrProf \\
\hline Chi-cuadrado & 7.202 & 27.221 & 8.675 & 4.823 & 14.694 & 18.025 & .801 & 3.329 & 10.924 & 6.385 & 10.433 & 6.374 \\
gl & 4 & 4 & 4 & 4 & 4 & 4 & 4 & 4 & 4 & 4 & 4 & 4 \\
Sig. asintót. & .126 & .000 & .070 & .306 & .005 & .001 & .938 & .504 & .027 & .172 & .034 & .173 \\
\hline
\end{tabular}

La prueba de Kruskal - Wallis permite aceptar H0, y por tanto indicar que no existen diferencias significativas entre la edad de los docentes y los factores, con un riesgo alfa de equivocarnos de $\mathrm{p}=.05$ o inferior en: PensCrit (7.202), BusqEfect (8.675), Socialización (4.823), Legalidad (0.801), EticaDig (3.329), ComprSocial (6.385) y DesarrProf (6.374). Por el contrario, si se rechaza la $\mathrm{H} 0$, y en consecuencia se acepta la $\mathrm{H} 1$, referida a la existencia de diferencias significativas a $\mathrm{p} \leq .05$ en los siguientes factores: Literacidad (27.221), Creación (14.694), Seguridad (18.825), IdentDig (10.924) y Autonomía (10.433).

Por lo que se refiere al grado académico, los valores alcanzados en el estadístico de Kolmogorov-Smirnov, se presentan en el cuadro $\mathrm{n}^{\mathbf{0}} 10$.

Saberes digitales en el profesorado universitario. Estudio en una escuela mexicana. José Alejandro Lara-Rivera y Julio Cabero-Almenara.

Página 11 de 19 
RED. Revista de Educación a Distancia. Núm. 66, Vol. 21. Artíc. 4, 30-Abril -2021

DOI: http://dx.doi.org/10.6018/red.447911

Cuadro 10. Prueba de normalidad (Grado) (Fuente: Elaboración propia).

\begin{tabular}{|c|c|c|c|c|c|c|c|c|}
\hline \multicolumn{9}{|c|}{ Pruebas de normalidad (Grado / Factores) } \\
\hline \multicolumn{9}{|c|}{ Kolmogorov-Smirnov } \\
\hline Factor & Grado & Sig. & Factor & Grado & Sig. & Factor & Grado & Sig. \\
\hline \multirow{3}{*}{ PensCrit } & Licenciatura & 0.002 & & Licenciatura & 0.004 & & Licenciatura & 0.005 \\
\hline & Maestría & 0.000 & Creacion & Maestría & 0.000 & IdenDig & Maestría & 0.002 \\
\hline & Doctorado & $.200^{\circ}$ & & Doctorado & 0.082 & & Doctorado & 0.002 \\
\hline \multirow{3}{*}{ Literacidad } & Licenciatura & 0.000 & & Licenciatura & 0.064 & & Licenciatura & 0.000 \\
\hline & Maestría & 0.000 & Seguridad & Maestría & 0.037 & ComprSocial & Maestría & 0.000 \\
\hline & Doctorado & 0.000 & & Doctorado & $.200^{\circ}$ & & Doctorado & 0.000 \\
\hline \multirow{3}{*}{ BusqEfect } & Licenciatura & 0.098 & & Licenciatura & 0.001 & & Licenciatura & 0.016 \\
\hline & Maestría & 0.004 & Legalidad & Maestría & 0.000 & Autonomia & Maestría & 0.004 \\
\hline & Doctorado & 0.086 & & Doctorado & 0.008 & & Doctorado & $.200^{\circ}$ \\
\hline \multirow{3}{*}{ Socializacion } & Licenciatura & 0.031 & & Licenciatura & 0.000 & & Licenciatura & $.200^{\circ}$ \\
\hline & Maestría & 0.017 & EticaDig & Maestría & 0.000 & DesarrProf & Maestría & 0.001 \\
\hline & Doctorado & 0.067 & & Doctorado & 0.000 & & Doctorado & 0.012 \\
\hline
\end{tabular}

Valores que de nuevo indican la no normalidad de la distribución, y en consecuencia se aplicó el estadístico de Kruskal - Wallis, alcanzándose los valores que se presentan en el cuadro no 11 .

Cuadro 11. Prueba de Kruskal-Wallis (Grado) (Fuente: Elaboración propia).

\begin{tabular}{|c|c|c|c|c|c|c|c|c|c|c|c|c|}
\hline & PensCrit & Literacidad & BusqEfect & Socializacion & Creacion & Seguridad & Legalidad & EticaDig & IdentDig & ComprSocial & Autonomia & DesarrProf \\
\hline Chi-cuadrado & 6.141 & .242 & 6.032 & 3.971 & 1.892 & 2.766 & .879 & 10.495 & 2.242 & 1.690 & 1.299 & 1.794 \\
\hline gl & 2 & 2 & 2 & 2 & 2 & 2 & 2 & 2 & 2 & 2 & 2 & 2 \\
\hline Sig. asintót. & .046 & .886 & .049 & .137 & .388 & .251 & .644 & .005 & .326 & .429 & .522 & .408 \\
\hline
\end{tabular}

La prueba de Kruskal - Wallis muestra que no se pueden rechazar las H0 con un riesgo alfa de $\mathrm{p}=.05$, o al menos en los siguientes factores: Literacidad (.242), Socialización (3.971), Creación (1.892), Seguridad (2.766), Legalidad (.879), IdentDig (2.242), ComprSocial (1.690), Autonomía (1.299) y DesarrProf (1.794). Por el contrario, si rechazamos la H0, y aceptamos en consecuencia la $\mathrm{H} 1$ con un riesgo alfa de equivocarnos de $\mathrm{p} \leq .05$, en los siguientes factores: PensCrit (6.141), BusqEfect (6.032) y EticaDig (10.495).

Finalmente, por lo que se refiere a la variable género, los valores obtenidos en el estadístico de Kolmogorov-Smirnov, para el análisis de la normalidad de la distribución se presentan en el cuadro $\mathrm{n}^{\circ} 12$.

Saberes digitales en el profesorado universitario. Estudio en una escuela mexicana. José Alejandro Lara-Rivera y Julio Cabero-Almenara. 
RED. Revista de Educación a Distancia. Núm. 66, Vol. 21. Artíc. 4, 30-Abril -2021

DOI: http://dx.doi.org/10.6018/red.447911

Cuadro 12. Prueba de normalidad (Género) (Fuente: Elaboración propia)

\begin{tabular}{|c|c|c|c|c|c|c|c|c|}
\hline \multicolumn{9}{|c|}{ Pruebas de normalidad (Género / Factores) } \\
\hline \multicolumn{9}{|c|}{ Kolmogorov-Smirnov } \\
\hline Factor & Género & Sig. & Factor & Género & Sig. & Factor & Género & Sig. \\
\hline PensCrit & $\begin{array}{l}\text { Hombre } \\
\text { Mujer }\end{array}$ & $\begin{array}{l}0.000 \\
0.000\end{array}$ & Creacion & $\begin{array}{c}\text { Hombre } \\
\text { Mujer }\end{array}$ & $\begin{array}{l}0.001 \\
0.001\end{array}$ & IdenDig & $\begin{array}{c}\text { Hombre } \\
\text { Mujer }\end{array}$ & $\begin{array}{l}0.000 \\
0.000\end{array}$ \\
\hline Literacidad & $\begin{array}{l}\text { Hombre } \\
\text { Mujer }\end{array}$ & $\begin{array}{l}0.000 \\
0.000\end{array}$ & Seguridad & $\begin{array}{c}\text { Hombre } \\
\text { Mujer }\end{array}$ & $\begin{array}{l}0.002 \\
.200^{*}\end{array}$ & ComprSocial & $\begin{array}{l}\text { Hombre } \\
\text { Mujer }\end{array}$ & $\begin{array}{l}0.000 \\
0.000\end{array}$ \\
\hline BusqEfect & $\begin{array}{l}\text { Hombre } \\
\text { Mujer }\end{array}$ & $\begin{array}{l}0.003 \\
0.008\end{array}$ & Legalidad & $\begin{array}{c}\text { Hombre } \\
\text { Mujer }\end{array}$ & $\begin{array}{l}0.000 \\
0.000\end{array}$ & Autonomia & $\begin{array}{l}\text { Hombre } \\
\text { Mujer }\end{array}$ & $\begin{array}{l}0.001 \\
0.031\end{array}$ \\
\hline Socializacion & $\begin{array}{l}\text { Hombre } \\
\text { Mujer }\end{array}$ & $\begin{array}{l}0.019 \\
0.040\end{array}$ & EticaDig & $\begin{array}{c}\text { Hombre } \\
\text { Mujer }\end{array}$ & $\begin{array}{l}0.000 \\
0.000\end{array}$ & DesarrProf & $\begin{array}{c}\text { Hombre } \\
\text { Mujer }\end{array}$ & $\begin{array}{l}0.003 \\
0.016\end{array}$ \\
\hline
\end{tabular}

También en este caso los datos mostraban la no normalidad de la distribución, aplicándose para el contraste de las H0 y H1, el estadístico U de Mann Whitney (Siegel, 1976). Los valores alcanzados se presentan en el cuadro $\mathrm{n}^{\circ} 13$.

Cuadro 13. Prueba U de Mann-Whitney (Género) (Fuente: Elaboración propia).

\begin{tabular}{|c|c|c|c|c|c|c|c|c|c|c|c|c|}
\hline & PensCrit & Literacidad & BusqEfect & Socializacion & Creacion & Seguridad & Legalidad & EticaDig & IdentDig & Comprsocial & Autonomia & Desarrprof \\
\hline Ude & 5883.500 & 5288.500 & 5787.500 & 18.000 & 6180.000 & 5869.500 & 5691.000 & 5028.000 & 5706.500 & 5390.500 & 6007.000 & 6052.500 \\
\hline W de W & 988.500 & 843.500 & 892.500 & 223.000 & 1735.000 & 12424.500 & 12246.000 & 1133.000 & 12261.500 & 11495.500 & 12562.000 & 12607.500 \\
\hline 2 & -.802 & -2.058 & -1.000 & -.314 & -.186 & -.827 & -1.202 & -2.634 & -1.168 & -3.110 & -.546 & -.451 \\
\hline Sig. asintót. (bilateral) & .423 & .040 & .317 & .753 & .852 & .408 & .229 & .008 & .243 & .002 & .585 & .652 \\
\hline
\end{tabular}

La prueba U de Mann-Whitney muestra que no existen diferencias significativas en el cruce de la variable Género al nivel de significación $\mathrm{p} \leq .05$ con los siguientes factores: PensCrit (802), BusqEfect (-1.000), Socialización (-.314), Creación (-.186), Seguridad (-.827), Legalidad (-1.202), IdentDig (-1.168), Autonomía (.546), y DesarrProf (-.451). La prueba U de Mann - Whitney nos muestra también que existen diferencias significativas en el cruce de la variable Género con los factores: Literacidad (-2.058), EticaDig (-2.634) y ComprSocial $(-3.110)$.

Para facilitar la comprensión de los resultados alcanzados, el cuadro $\mathrm{n}^{\mathbf{0}}$ 14, muestra la matriz de intersecciones entre los factores y las variables de cruce. Observamos que de las 36 posibilidades $(12 \times 3)$, existen 11 combinaciones con diferencias significativas, siendo la EDAD de los profesores la variable que más se repite con 5, seguido del Grado Académico y el Género con 3 cada una.

Saberes digitales en el profesorado universitario. Estudio en una escuela mexicana. José Alejandro Lara-Rivera y Julio Cabero-Almenara. 
RED. Revista de Educación a Distancia. Núm. 66, Vol. 21. Artíc. 4, 30-Abril -2021

DOI: http://dx.doi.org/10.6018/red.447911

Cuadro 14. Intersecciones (Variables / 12 Factores). Nota: 0.- No existen diferencias significativas y $1=$ Existen diferencias significativas (Fuente: Elaboración propia).

\begin{tabular}{|l|c|c|c|}
\cline { 2 - 4 } \multicolumn{1}{c|}{} & \multicolumn{3}{c|}{ Variables } \\
\hline Factores & Edad & Grado & Género \\
\hline Pensamiento crítico & 0 & 1 & 0 \\
\hline Literacidad & 1 & 0 & 1 \\
\hline Búsqueda efectiva & 0 & 1 & 0 \\
\hline Socialización & 0 & 0 & 0 \\
\hline Creación & 1 & 0 & 0 \\
\hline Seguridad & 1 & 0 & 0 \\
\hline Legalidad & 0 & 0 & 0 \\
\hline Ética digital & 0 & 1 & 1 \\
\hline Identidad digital & 1 & 0 & 0 \\
\hline Compromiso social & 0 & 0 & 1 \\
\hline Autonomía & 1 & 0 & 0 \\
\hline Desarrollo profesional & 0 & 0 & 0 \\
\hline Dif. Sig. $=$ & $\mathbf{5}$ & $\mathbf{3}$ & $\mathbf{3}$ \\
\hline
\end{tabular}

Al ser la variable EDAD la de mayor incidencia en los resultados, se realizó un análisis intra grupo para identificar el comportamiento hacia el interior, los resultados se presentan en el cuadro $\mathrm{n}^{\circ} 15$.

Cuadro 12. Análisis intra grupo de la variable EDAD. (Fuente: Elaboración propia).

\begin{tabular}{|c|c|c|c|c|c|c|}
\hline \multicolumn{7}{|c|}{ Edad - Saberes Digitales } \\
\hline Edad & Sujetos & $\begin{array}{c}\text { Valor máx } \\
\text { item }\end{array}$ & $\begin{array}{c}\text { No. de } \\
\text { items }\end{array}$ & $\begin{array}{c}\text { Valor Máx } \\
\text { esperado }\end{array}$ & $\begin{array}{c}\text { Valor } \\
\text { Obtenido }\end{array}$ & $\%$ Obtenido \\
\hline $24-29$ & 19 & 5 & 59 & 5,605 & 4,567 & $81.5 \%$ \\
\hline $30-39$ & 68 & 5 & 59 & 20,060 & 16,439 & $81.9 \%$ \\
\hline $40-49$ & 66 & 5 & 59 & 19,470 & 15,107 & $77.6 \%$ \\
\hline $50-59$ & 52 & 5 & 59 & 15,340 & 11,752 & $76.6 \%$ \\
\hline $60-70$ & 19 & 5 & 59 & 5,605 & 4,357 & $77.7 \%$ \\
\hline
\end{tabular}

Observamos que los grupos que obtuvieron el mayor puntaje fueron: 30-39 años (81.9\%) y 24-29 años (81.5\%), y estos resultados marcan diferencia positiva con los profesores de mayor edad. En la gráfica $n^{\circ} 2$, se muestran los resultados para cada bloque de edad y el porcentaje obtenido.

Saberes digitales en el profesorado universitario. Estudio en una escuela mexicana. José Alejandro Lara-Rivera y Julio Cabero-Almenara. 
Gráfica 2. Resultado de los saberes digitales y la variable Edad significativas (Fuente: Elaboración propia).

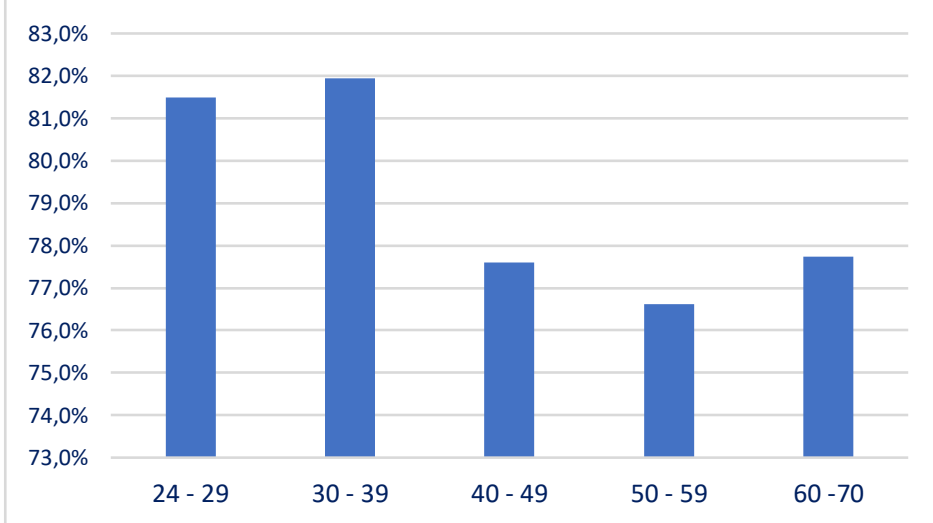

\section{Conclusiones}

Las conclusiones de la investigación van en diferentes direcciones. En primer lugar, en lo referido al instrumento de investigación elaborado para el diagnóstico de los saberes digitales de los profesores. Herramienta que respecto a otras utilizadas incorpora otras dimensiones y factores (pensamiento crítico, literacidad, búsqueda efectiva, ...) que deben empezar a contemplarse para el análisis y conocimiento de las actitudes que los docentes presentan ante las tecnologías de la información. Ello nos lleva a la necesidad de repensar los diferentes marcos de competencias digitales formulados (Cabero, Romero, Barroso \& Palacios, 2020). De igual manera es importante señalar los altos valores encontrados, en lo que respecta a los índices de fiabilidad del instrumento y la adecuación de los ítems a las diferentes dimensiones propuestas.

Los resultados obtenidos en la variable de cruce Edad, mostraron diferencias significativas en cinco factores; literacidad, creación de contenido digital, seguridad digital, identidad digital y autonomía. En conjunto, estos factores abarcan un total de 26 ítems, sin embargo, haremos especial referencia a 7 de ellos, que a nuestro criterio son de gran relevancia y es en donde se podrían focalizar los esfuerzos en el corto plazo para disminuir la brecha digital entre los docentes de menor y mayor edad.

Ítems de corte Actitudinal:

- Dar significado al currículum escolar identificando aprendizajes posibles de desarrollar con la incorporación de herramientas tecnológicas.

- Identificación clara sobre las fortalezas y debilidades de las TIC en la actividad docente.

- Concientización respecto a la necesidad de formación en herramientas digitales aplicadas a la educación superior.

Ítems de corte Instrumental:

Saberes digitales en el profesorado universitario. Estudio en una escuela mexicana. 
- Manejo de paquetería Office (Word, Excel, PowerPoint).

- Creación de tutoriales en plataformas como YouTube.

- Elaboración de materiales y recursos educativos digitales.

- Aprendizaje autónomo sobre el uso de herramientas digitales.

Los hallazgos coinciden con los resultados obtenidos por otros investigadores (GallardoEchenique, Poma \& Esteve, 2018; Flores-Lueg \& Roig-Vila, 2019; López, Pozo \& Fuentes, 2019; Pozo, López, Fernández \& López, 2020) que ponen de manifiesto que los docentes de mayor edad presentan más problemas para la incorporación de las tecnologías a las prácticas educativas que los más jóvenes.

La investigación permite obtener un diagnóstico preliminar sobre las TIC en la práctica docente de la Universidad Autónoma de Occidente, donde ha quedado de manifiesto que la edad de los profesores es un factor diferenciador en dos vías: actitudinal e instrumental. Consideramos necesario realizar indagaciones de corte cualitativo para formular conclusiones más detalladas. Es importante que los profesores estén convencidos y comprometidos con la inclusión de la tecnología en su práctica docente y transiten hacia nuevas metodologías que respondan a la problemática actual. Aunque de todas formas es necesaria su replicación en otros contextos para acrecentar, por una parte, la validez del instrumento de forma general y de las diferentes dimensiones contrastadas de manera particular.

También pudiera ser conveniente que las nuevas dimensiones contempladas en el estudio se incorporen a instrumentos anteriormente utilizados en el análisis de las competencias digitales docentes, y a través de análisis exploratorios y confirmatorios, analizar la viabilidad de su incorporación. Ello lleva a nuevos retos investigadores.

Presentación del artículo: 29 de septiembre de 2020 Fecha de aprobación: 20 de febrero de 2021

Fecha de publicación: 30 de abril de 2021

Lara-Rivera, J. y Cabero-Almenara, J. (2021). Saberes digitales en el profesorado universitario. Estudio en una escuela mexicana. RED. Revista de educación a distancia, 21(66). http://dx.doi.org/10.6018/red.447911

\section{Financiación}

El proyecto fue financiado por la Secretaría de Educación Pública de México, a través del Programa para el desarrollo profesional docente, para el tipo superior (PRODEP). Folio: UDO-EXB-203. 


\section{Referencias bibliográficas}

Cabero, J. (2015). Tendencias para el aprendizaje digital: de los contenidos cerrados al diseño de materiales centrado en las actividades. El Proyecto Dipro 2.0. Revista de Educación a Distancia (RED), (32). https://revistas.um.es/red/article/view/233041

Cabero, J. (2014). La formación del profesorado en TIC: Modelo TPACK (Conocimiento Tecnológico, Pedagógico y de Contenido). Revista Sevilla: Secretariado de Recursos Audiovisuales y Nuevas Tecnologías de la Universidad de Sevilla.

Cabero, J. (2020). Aprendiendo del tiempo de la COVID-19. Revista electrónica Educare. EISSN: 1409-4258 Vol. 24, Suplemento Especial, 2020: 1-3.

Cabero, J. (dir.) (2014). La formación del profesorado en TIC: Modelo TPACK. Píxel-Bit. Revista de Medios y Educación, (46), 253-254.

Cabero, J. y Martínez, A. (2019). Las tecnologías de la información y comunicación y la formación inicial de los docentes: modelos y competencias digitales. Profesorado: Revista de Currículum y Formación de Profesorado, 23 (3), 247-268.

Cabero, J., y Palacios, A. (2020). Marco Europeo de Competencia Digital Docente «DigCompEdu» y cuestionario «DigCompEdu Check-In». EDMETIC. Revista de Educación Mediática y TIC, 9(1), 213-234. https://doi.org/10.21071/edmetic.v9i1.12462

Cabero, J., Romero, R., Barroso, L. y Palacios, A. (2020). Marcos de competencias digitales docentes y su adecuación al profesorado universitario y no universitario. Revista Caribeña de Investigación Educativa, (RECIE), 4(2), 137-158.

Cai, Z., Fan, X., y Du, J. (2017). Gender and attitudes toward technology use: A metaanalysis. Computers \& Education, 105, 1-13. https://doi.org/10.1016/j.compedu.2016.11.003.

Cuban, L. (2001). Oversold \& underused: Computers in the classroom: Harvard University Press Cambridge, Massachusetts London, England.

Del Valle, S. et. al. (2016). La actitud de los docentes frente a las tecnologías de la información y comunicación. Cuadernos de la facultad de humanidades y ciencias sociales de la Universidad Nacional de Jujuy, 50, pp. 120-134.

Durán, M., Gutiérrez, I., y Prendes, M.P. (2016). Certificación de la competencia TIC del profesorado universitario: Diseño y validación de un instrumento. Revista mexicana de investigación educativa, 21(69), 527-556. 
Fernández-Cruz, F. J., y Fernández-Díaz, M. J. (2016). Los docentes de la generación Z y sus competencias digitales. Generation Z's teachers and their digital skills. Revista Comunicar, 24(46), 97-105. https://doi.org/10.3916/C46-2016-10.

Flores-Lueg, C. y Roig-Vila, R. (2019). Factores personales que inciden en la autovaloración de futuros maestros sobre la dimensión pedagógica del uso de TIC. Revista Iberoamericana de Educación Superior, 27(X), 151-171, doi: dx.doi.org/10.22201/iisue.20072872e.2019.27.345

Gallardo, E., Poma, A. y Esteve, F. (2018). La competencia digital: análisis de una experiencia en el contexto universitario. Academicus, 12(1), 6-15.

Garzón, E., Sola, T., Ortega, J., Marín, J., y Gómez, G. (2020). Teacher Training in Lifelong Learning. The Importance of Digital Competence in the Encouragement of Teaching Innovation. Sustainability, 12, 28-52. https://doi.org/10.3390/su12072852

González, M. y Abad, E. (2020). El aula invertida: un desafío para la enseñanza universitaria Virtualidad, Educación y Ciencia, 20(11), pp. 75-91.

Grijalva, A., y Moreno, D. (2017). Empoderamiento social en contextos violentos mexicanos mediante la competencia mediática. Comunicar, 25(53), 29-38.

Grijalva-Verdugo, A. y Lara-Rivera, J. (2019). Competencia mediática en jóvenes universitarios. Análisis de saberes para producir contenido digital en una IES mexicana. Revista Edutec, No. 67, pp. 16-30.

Hatlevik, O. E., Throndsen, I., Loi, M., y Gudmundsdottir, G. B. (2018). Students' ICT selfefficacy and computer and information literacy: Determinants and relationships. $\begin{array}{llll}\text { Computers } & \mathrm{y} & \text { Education, } & 118,\end{array}$ https://doi.org/10.1016/J.COMPEDU.2017.11.011

Hernández y Hernández, D., Ramírez-Martinell, A., y Cassany, D. (2014). Categorizando a los usuarios de sistemas digitales. Píxel-Bit. Revista De Medios Y Educación, (44), 113126. https://doi.org/10.12795/pixelbit.2014.i44.08

Infante-Moro, A., Infante-Moro, J. C., y Gallardo-Pérez, J. (2021). La adquisición de competencias TIC en el ámbito universitario: el caso de la Facultad de Ciencias Empresariales y Turismo de la Universidad de Huelva. Pixel-Bit. Revista de Medios y Educación, 60,29-58. https://doi.org/10.12795/pixelbit.79471

Lara, J. y Grijalva, A. (2018). E-ciudadanía y Educación Universitaria. Evaluación de Saberes Digitales en una IES mexicana. Revista Eticanet, 2 (18), pp. 298-315.

Lázaro, J. L., Usart, M., y Gisbert, M. (2019). Assessing Teacher Digital Competence: the Construction of an Instrument for Measuring the Knowledge of Pre-Service Teachers. 
RED. Revista de Educación a Distancia. Núm. 66, Vol. 21. Artíc. 4, 30-Abril -2021

DOI: http://dx.doi.org/10.6018/red.447911

Journal of New Approaches in Educational Research, 8(1), 73-78. doi:10.7821/naer.2019.1.370.

López, J.A., López, J., Moreno, A.J. y Pozo, S. (2020). Efectiveness of Innovate Educational Practices with Flipped Learning and Remote Sensing in Earth and Environmental Sciences-An Exploratory Case Study. Remote Sens. $\quad 12, \quad 897$; doi:10.3390/rs12050897.

López, J., Pozo, S., Fuentes, C. y Romero, J.M. (2019). Análisis del Liderazgo Electrónico y la Competencia Digital del Profesorado de Cooperativas Educativas de Andalucía (España). Multidisciplinary Journal of Educational Research, 9(2), 194-223. http://10.4471/remie.2019.4149.

Mateo, J. (2004). La investigación ex post-facto. En R. Bisquerra (coord.). Metodología de la investigación educativa (pp. 195-230). Madrid: la Muralla.

Rodríguez-García, A., Raso, F., y Ruiz-Palmero, J. (2019). Competencia digital, educación superior y formación del profesorado: un estudio de meta-análisis en la web of science. Pixel-Bit, 54(4), 65-81. https://doi.org/10.12795/pixelbit.2019.i54.04.

Roig-Vila, R., Mengual-Andrés, S., y Quinto-Medrano, P. (2015). Conocimientos tecnológicos, pedagógicos y disciplinares del profesorado de Primaria. Comunicar, 45(23), 151-159. https://doi.org/http://dx.doi.org/10.3916/C45-2015-16

Sáez, J. (2010). Actitudes de los docentes respecto a las TIC, a partir del desarrollo de una práctica reflexiva. Revista Escuela Abierta: e-ISNN: 2603-588X, España.

Ruiz-Cabezas, A., Medina, M., Pérez, E. y Media, A (2020). University teachers' training: The Digital Competence. [Formación del profesorado Universitario en la Competencia Digital]. Pixel-Bit. Revista de Medios y Educación, 58, 181-215. https://doi.org/10.12795/pixelbit.74676

Sansot, S. (2020). Construcción de saberes didácticos mediados por tecnologías digitales: posibilidades y límites de prácticas de enseñanza situadas en profesorados universitarios. Revista Virtualidad, Educación y Ciencia. No. 21 (11), pp. 70-83. ISSN: $1853-6530$.

Siddiq, F., y Scherer, R. (2019). Is there a gender gap? A meta-analysis of the gender differences in students' ICT literacy. Educational Research Review, 27, 205-217.

Siegel, S. (1976). Estadística no paramétrica. México: Trillas.

Silva, J., Lázaro, J.L., Miranda, P., Morales, M. J., Gisbert, M., Rivoir, A., y Onetto, A. (2019). La Competencia Digital Docente en Formación Inicial: Estudio a Partir de los Casos de Chile y Uruguay. Education Policy Analysis Archives, 27.

Saberes digitales en el profesorado universitario. Estudio en una escuela mexicana. José Alejandro Lara-Rivera y Julio Cabero-Almenara.

Página 19 de 19 
Solís, J. y Jara, V. (2019). Competencia digital de docentes de ciencias de la salud de una universidad chilena. Pixel-Bit. Revista de Medios y Educación, 56, 193-211. https://doi.org/10.12795/pixelbit.2019.i56.10.

Uerz, D., Volman, M. y Kral, M. (2018). Teacher educators' competences in fostering student teachers' proficiency in teaching and learning with technology: An overview of relevant research literature. Teaching and Teacher Education, 70, 12-23. https://doi.org/10.1016/j.tate.2017.11.005.

UNESCO (2016). Competencias y estándares TIC desde la dimensión pedagógica. Una perspectiva desde los niveles de apropiación de las TIC en la práctica docente. Pontificia Universidad Javeriana - Cali. 